\title{
Implication of Existence of Hybrid stars and Theoretical Expectation of Submillisecond Pulsars
}

\author{
Xiaoping Zheng ${ }^{\mathrm{a}, *}$, Nana Pan ${ }^{\mathrm{a}}$, Shuhua Yang ${ }^{\mathrm{a}}$, Xuewen Liu ${ }^{\mathrm{a}}$, \\ Miao Kang ${ }^{a}$ and Jiarong $\mathrm{Li}^{\mathrm{b}}$ \\ ${ }^{a}$ Institute of Astrophysics, Huazhong Normal University, Wuhan 430079, P. R. \\ China \\ ${ }^{\mathrm{b}}$ Institute of Particle Physics, Huazhong Normal University, Wuhan 430079, P. R. \\ China
}

\begin{abstract}
We derive the bulk viscous damping timescale of hybrid stars, neutron stars with quark matter core. The r-mode instability windows of the stars show that the theoretical results are consistent with the rapid rotation pulsar data, which may give an indication for the existence of quark matter in the interior of neutron stars. Hybrid stars instead of neutron or strange stars may lead to submillisecond pulsars.
\end{abstract}

Key words: dense matter — gravitation — stars: neutron — stars:rotation stars:oscillations

PACS: 97.60.Jd, 12.38.Mh, 97.60.Gb

Probing the composition of matter in the interior of compact stars is of interest for understanding of pulsars. The available theories have not yet given a unique solution to this problem. Since the deconfinement transition from hadron matter into quark matter is possible at high density, one logically proposes that some pulsars could be strange stars. But how to distinguish strange stars from neutron stars is a difficult and an urgent issue. First, one suggested that bulk properties of neutron stars such as radius and moment of inertia, may be marked off due to the difference between self-bound and gravity-bound matter. Unfortunately, the bulk properties of these two kinds of stars are similar in the observed mass range $1<M / M_{\odot}<2$ (Haensel. Zdunik and Schaeffer, 1986). Another way is to look at the possible difference of their highest rotation

* corresponding author.

Email address: zhxp@phy.ccnu.edu.cn (Xiaoping Zheng). 
frequency, since the discovery by Andersson, Friedman and Morsink of r-mode instabilities in the rotating compact stars put rather severe limits on the highest rotation frequency of pulsars (Andersson, 1998; Friedman and Morsink, 1998). A pioneer investigation found that the limiting rotation of strange stars is in millisecond period range but for normal neutron stars in the period range of 15 20ms (Madsen, 1998, 2000). However, a strange star evolving to the limiting frequency corresponds to temperature in $10^{6}--10^{7} \mathrm{~K}$ range instead of the inferred core temperature of millisecond pulsars of a few times $10^{8} \mathrm{~K}$. Obviously, this is a serious drawback if we attributed the uncorrespondence to the under-representing statistics due to a low number of objects as suggested by Madsen(Madsen, 2000). Although some works also argued that neutron stars with solid crusts would be the candidates to explain the rapidly rotating accreting pulsar's data through thermal runaway recycle of r-mode. Due to the presence of viscous boundary layer damping(Bildsten and Ushomirsky, 2000; Andersson et al., 2000), the resulting viscous heating is so intense that it can heat the crust-core interface to the melting temperature of the solid crust when the r-mode amplitude is larger than some critical value, which is crudely estimated to be $\sim 10^{-3}$ by Owen (1999) and a more accurate value is given as $5 \times 10^{-3}$ by (Lindblom, Owen and Ushomirsky, 2000).

Following the above train of thought, we will here investigate the r-mode instability window of hybrid stars, i.e., the neutron stars containing quark matter core. We show that the instability window of hybrid stars explains the millisecond pulsar data very well, but the strange stars and normal neutron stars would not fit because of the weaknesses concerned above.

In rotating relativistic stars, gravitational radiation drives the r-mode while various dissipation mechanisms counteract the fluid motion. In general, shear viscosity, or surface rubbing that could also be applied to the hybrid stars, which also have a solid crust similar to the normal neutron stars, suppresses the mode at low temperatures but bulk viscosity dominates at high temperatures. The critical rotation frequency for a given stellar model as a function of temperature follows

$$
\frac{1}{\tau_{g r}}+\frac{1}{\tau_{s v}}+\frac{1}{\tau_{b v}}+\frac{1}{\tau_{s r}}=0,
$$

where $\tau_{g r}<0$ is the characteristic timescale for energy loss due to gravity wave emission, $\tau_{s v}$ and $\tau_{b v}$ are the damping times due to shear and bulk viscosities, and $\tau_{s r}$ is that for surface rubbing due to the presence of the viscous boundary layer.

A polytropic equation of state with a low index $n$ is a good approximation for compact stars, as discussed in many papers (Kokkotas and Stergioulas, 1999; Lindblom, Mendell and Owen, 1999). The timescale for $n=1$ polytrope from 
gravity wave emission can be written as

$$
\tau_{\mathrm{gr}}=-47 \mathrm{~s} \times\left(\frac{M}{1.4 M_{\odot}}\right)^{-1}\left(\frac{R}{10 \mathrm{~km}}\right)^{-4}\left(\frac{1000}{\nu}\right)^{6},
$$

where $M, R, \nu$ represent the mass, radius and the rotation frequency of the star respectively. In general, the shear viscosity and surface rubbing dominate at low temperature, but in fact we can neglect the shear viscosity because the dissipation associated with a viscous boundary layer would greatly exceed that of the standard shear viscosity by typical $10^{5}$ (Bildsten and Ushomirsky, 2000; Andersson et al., 2000). The timescale due to surface rubbing is given by (Andersson et al., 2000)

$$
\tau_{s r}=200 \mathrm{~s} \times\left(\frac{M}{1.4 M_{\odot}}\right)\left(\frac{R}{10 \mathrm{~km}}\right)^{-2}\left(\frac{T}{10^{8} \mathrm{~K}}\right)\left(\frac{1000}{\nu}\right)^{1 / 2} .
$$

We now discuss the bulk viscosity coefficients of strange quark matter, hadron matter and their mixed phase matter to estimate the bulk viscous damping timescale in hybrid stars. The bulk viscosities are given approximately by

$$
\zeta_{Q P}=3.2 \times 10^{21} \frac{\rho T_{9}^{2}}{\kappa^{2} \Omega^{2}}\left(\frac{m_{s}}{100 \mathrm{Mev}}\right)^{4}
$$

for strange quark matter(Madsen, 1992), and

$$
\zeta_{H P}=6 \times 10^{-5} \frac{\rho^{2} T_{9}^{6}}{\kappa^{2} \Omega^{2}}
$$

for hadron matter (Ipser and Lindblom, 1991), where the constant $\kappa=2 / 3$, the angular velocity $\Omega=2 \pi \nu$ and $\rho$ is the density, $m_{s}$ is strange quark mass. To construct hybrid stars, we consider the deconfinement transition occurring at high density inside the compact stars and use BPS(Baym. Pethick and Sutherland, 1971) and GPS(Ghosh, Phatak and Sahu, 1995) equations of state for hadrons and MIT model for quarks. As in most cases, the variation of volume per unit mass and the chemical potential imbalance due to the oscillation in the star is so small that it is the liner part that contributes most to the viscosity, so we have used the relaxation time approximation method(Lindblom and Owen, 2002; Drago, Lavagno and Pagliara, 2005; Nayyar and Owen, 2005) to compute the bulk viscosity of the mixed phase matter,

$$
\zeta=\frac{P\left(\gamma_{\infty}-\gamma_{0}\right) \tau}{1+(\omega \tau)^{2}}
$$




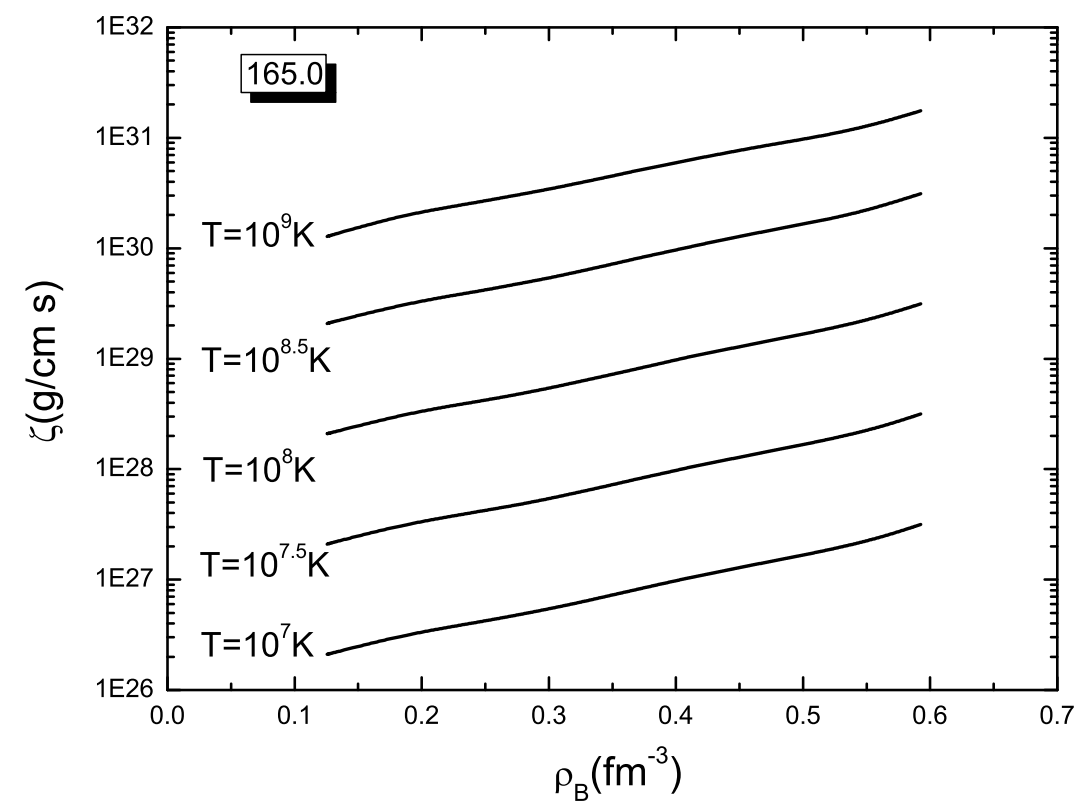

Fig. 1. The bulk viscosity of the mixed phase matter in the hybrid star for $m_{s}=150 \mathrm{Mev}$ and $B^{1 / 4}=165 \mathrm{Mev}$ in terms of GPS model(Ghosh, Phatak and Sahu, 1995).

Here $P$ is the pressure of the mixed phase, $\gamma_{\infty}$ and $\gamma_{0}$ are the "infinite" and "zero" frequency adiabatic index, $\omega$ is the angular velocity of the perturbation and $\tau$ is the relaxation time associated to the weak processes. As an example, we numerically calculate the bulk viscosity coefficient of the mixed phase matter which is showed in figure 1 for bag constant $B^{1 / 4}=165 \mathrm{Mev}$. From numerical estimate, we easily find the fact that $\zeta_{Q P} \approx \zeta_{M P} \gg \zeta_{H P}$.

Now, we can use the bulk viscosities listed above to evaluate the bulk viscous damping times for the hybrid stars. For the given energy of modes $E$ and its variation rate $\dot{E} \equiv \mathrm{d} E / \mathrm{d} t$, the timescale is defined as

$$
\frac{1}{\tau}=-\frac{\dot{E}}{2 E}
$$

The r-mode energy in a rotating relativistic star with radius $R$ has been expressed as(Lindblom, Mendell and Owen, 1999)

$$
E=\frac{\alpha^{2} \pi}{2 m}(m+1)^{3}(2 m+1) ! R^{4} \Omega^{2} \int_{0}^{R} \rho(r)\left(\frac{r}{R}\right)^{2 m+2} \mathrm{~d} r+O\left(\Omega^{4}\right)
$$


where $\alpha$ is the normalization parameter, $m$ is integer and $\rho(r)$ is the density function(Andersson and Kokkotas, 2001). The energy dissipation rate due to bulk viscosity can be estimated with the following formula

$$
\dot{E}_{b v}=-\int \zeta \delta \sigma \delta \sigma^{*} \mathrm{~d}^{3} x
$$

where $\delta \sigma$ is expansion tensor of Eulerian perturbation of velocity field, expressed approximately as(Lindblom, Mendell and Owen, 1999)

$$
\delta \sigma=-i(\omega+m \Omega) \frac{\delta \rho}{\rho}
$$

here $\omega$ denotes angular frequency of the r-mode. In lowest-order approximation, we easily find $\delta \sigma \sim R^{2}\left(\frac{r}{R}\right)^{m+1}$.In a stratiform star, we can decompose the energy $E$ into four terms, i.e., the quark core, mixed phase, nuclei envelope and solid crust denoted respectively by $Q P, M P, H P$ and $C R$

$$
E=\left(\frac{R_{Q P}}{R}\right)^{2 m-2}\left(\tilde{E}_{Q P}+\tilde{E}_{M P}+\tilde{E}_{H P}+\tilde{E}_{C R}\right)
$$

with

$$
\begin{aligned}
& \tilde{E}_{Q P}=\frac{\alpha^{2} \pi}{2 m}(m+1)^{3}(2 m+1) ! R_{Q P}^{4} \Omega^{2} \int_{0}^{R_{Q P}} \rho_{Q P}(r)\left(\frac{r}{R_{Q P}}\right)^{2 m+2} \mathrm{~d} r \\
& \tilde{E}_{M P}=\frac{\alpha^{2} \pi}{2 m}(m+1)^{3}(2 m+1) ! R_{Q P}^{4} \Omega^{2} \int_{R_{Q P}}^{R_{M P}} \rho_{M P}(r)\left(\frac{r}{R_{Q P}}\right)^{2 m+2} \mathrm{~d} r \\
& \tilde{E}_{H P}=\frac{\alpha^{2} \pi}{2 m}(m+1)^{3}(2 m+1) ! R_{Q P}^{4} \Omega^{2} \int_{R_{M P}}^{R_{H P}} \rho_{H P}(r)\left(\frac{r}{R_{Q P}}\right)^{2 m+2} \mathrm{~d} r \\
& \tilde{E}_{C R}=\frac{\alpha^{2} \pi}{2 m}(m+1)^{3}(2 m+1) ! R_{Q P}^{4} \Omega^{2} \int_{R_{H P}}^{R} \rho_{C R}(r)\left(\frac{r}{R_{Q P}}\right)^{2 m+2} \mathrm{~d} r .
\end{aligned}
$$

A constant density in each phase, replaced by corresponding mean density, is a good approximation in terms of our calculations of the stellar structure(see figure 2). Considering $\zeta_{Q P, M P} \gg \zeta_{H P, C R}$, we have, according to (8) and (9),

$$
\dot{E}_{b v}=\left(\frac{R_{Q P}}{R}\right)^{2 m-2}\left(\dot{E}_{Q P}+\dot{E}_{M P}\right)
$$




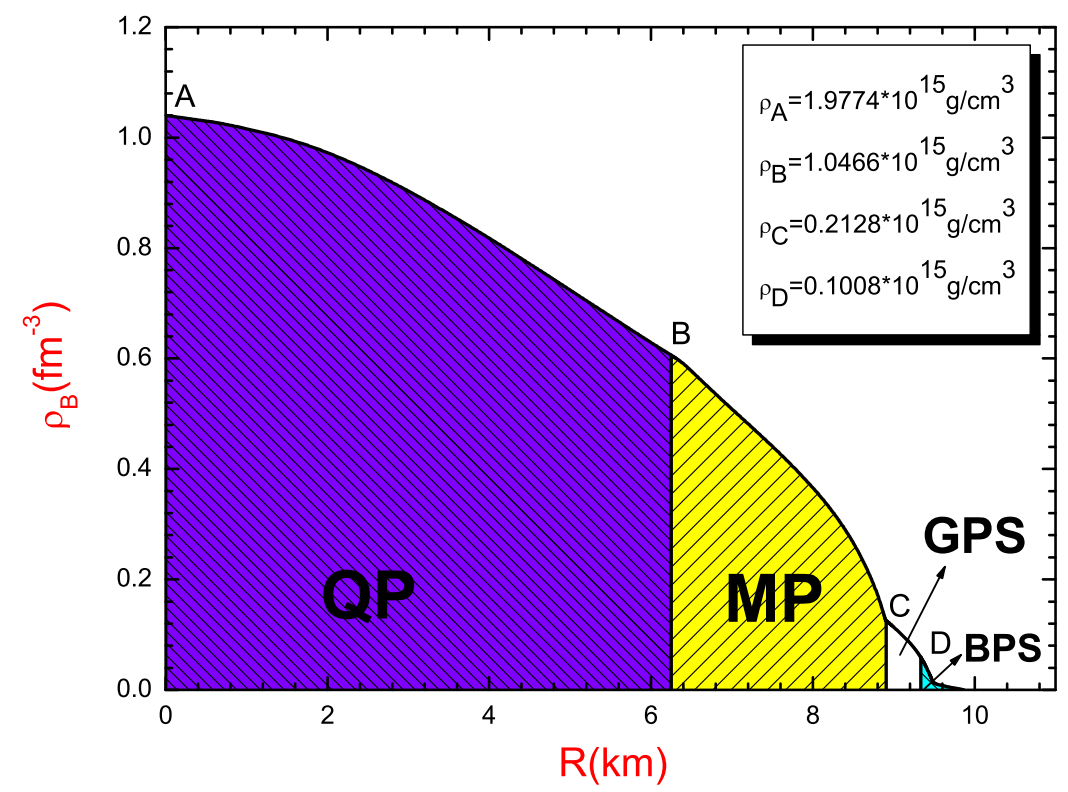

Fig. 2. The stratiform structure of the hybrid star with parameters $M=1.4 M_{\odot}$, $R=9.87 \mathrm{~km}, m_{s}=150 \mathrm{Mev}$ and $B^{1 / 4}=165 \mathrm{Mev}$ in terms of GPS model(Ghosh, Phatak and Sahu, 1995). Here QP and MP represent the quark phase and mixed phase in the hybrid star respectively.

with

$$
\begin{aligned}
& \dot{E}_{Q P}=\left(\frac{d E}{d t}\right)_{R_{Q P}, \rho_{Q P}}, \\
& \dot{E}_{M P}=\left(\frac{R_{M P}}{R_{Q P}}\right)^{2 m-2}\left(\frac{d E}{d t}\right)_{R_{M P}, \rho_{M P}}-\left(\frac{d E}{d t}\right)_{R_{Q P}, \rho_{M P}} .
\end{aligned}
$$

Thus, the timescale from $\mathrm{Eq}(4)$ is immediately expressed as

$$
\tau_{b v}=A \tau_{b v}^{Q P}(R)
$$

where $A$ is a constant related to the stellar structure that is showed in table $1, \tau_{b v}^{Q P}(R)$ can be given by (Andersson and Kokkotas, 2001)

$$
\tau_{b v}^{Q P}=7.9 \mathrm{~s} \times\left(\frac{M}{1.4 M_{\odot}}\right)^{2}\left(\frac{R}{10 \mathrm{~km}}\right)^{-4}\left(\frac{T}{10^{9} \mathrm{~K}}\right)^{-2}\left(\frac{m_{s}}{100 \mathrm{MeV}}\right)^{-4}\left(\frac{1000}{\nu}\right)^{2} .
$$

Inserting (2), (3) and (18), we apply Eq(1) to evaluate the critical frequency of 
Table 1

The Values of $A$ for different Structures of a Hybrid Star with $M=1.4 M_{\odot}$

\begin{tabular}{clc}
$B^{1 / 4}[\mathrm{MeV}]$ & $R[\mathrm{~km}]$ & $A$ \\
\hline 165.0 & 9.87 & 0.6886 \\
175.0 & 10.41 & 0.6929 \\
185.0 & 12.67 & 6.5488 \\
\hline
\end{tabular}

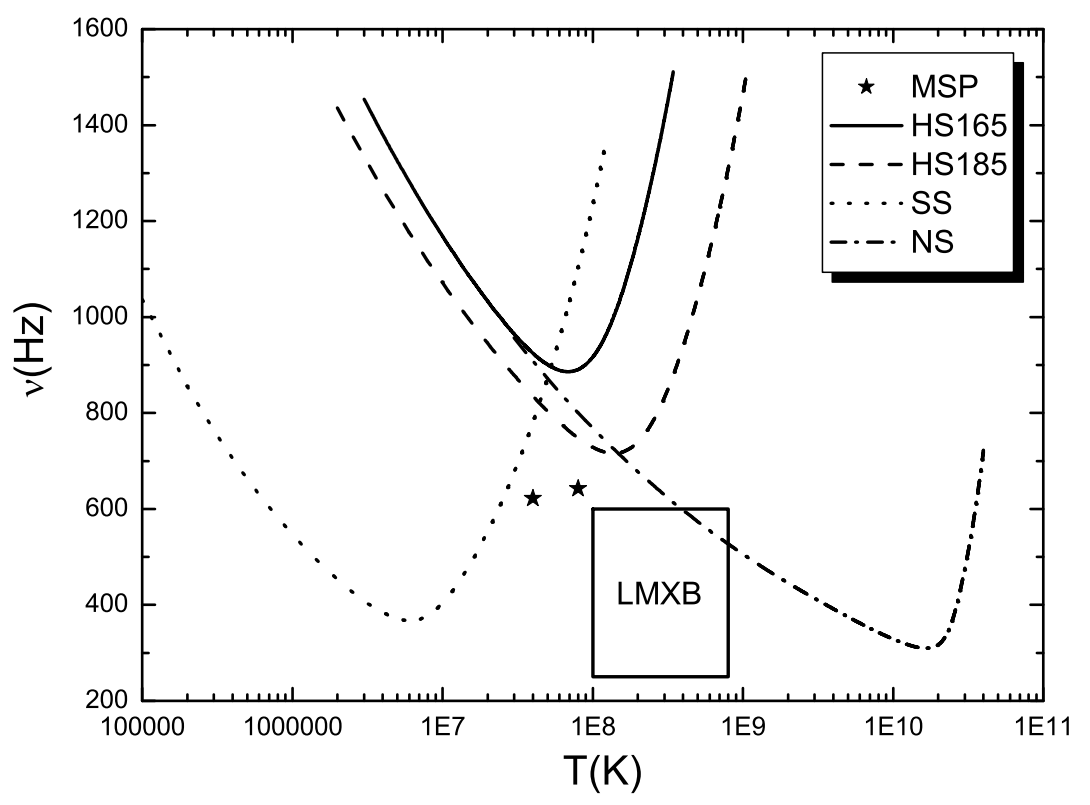

Fig. 3. Comparison of r-mode instability windows of hybrid stars with several other models(Andersson et al., 2000; Madsen, 2000; Zheng et al., 2003). HS, SS and NS denote hybrid star, strange star and neutron star respectively, while the star symbol and the rectangle indicate the two observed fastest pulsars and the Low Mass X-rays Binaries.

the rotating star as a function of temperature. Figure 3 shows the instability windows of the hybrid stars for $M=1.4 M_{\odot}$ and $R$ ranging from $10 \sim 14 \mathrm{~km}$. As comparison, we also give the windows of strange star and normal star in figure 3 .

For hybrid stars, one notes that a pulsar will reach the frequency of about $700 \mathrm{~Hz}(1.43 \mathrm{~ms})$ following a track coincident with the curve marking the instability region. So, the LMXB's are well within the region stable against r-mode instabilities, just spinning up the region unhindered by the instability due to accretion. For strange stars, there is the same mechanism as hybrid star model but a deviation of temperature from the pulsar data(Madsen, 2000; 
Zheng et al., 2003). It is accepted that the neutron stars with crust could also fit the pulsar data through r-mode runaway recycle mechanism (Levin, 1999; Bildsten and Ushomirsky, 2000; Andersson et al., 2000). An important point is that, the fate of the crust of neutron stars is different from that of hybrid stars: the former is melted probably because of r-mode dissipation due to viscous boundary layer. We can imagine that the existence of the higher rotation pulsars is possible if there would exist larger quark matter core in the interior of a compact star. This may imply that submillisecond pulsars, if they exist, should be hybrid stars instead of neutron stars or strange stars.

In conclusion, the bulk viscous damping timescale for hybrid stars is estimated for a range of parameters through which the r-mode instability window is obtained. The rapid rotation pulsar data is a significant indication of the existence of hybrid stars instead of neutron stars or strange stars. Naturally, this is a possible evidence of quark matter in the interior of the compact stars.

We would like to thank the support by National Natural Science Foundation of China under Grant No. 10373007, 90303007 and the Ministry of Education of China with project No. 704035. We are especially grateful to Professor D.F. Hou for reading and checking the text.

\section{References}

Andersson, N., 1998, ApJ, 502, 708.

Andersson, N., Jones, D.I., Kokkotas, K.D. \& Stergioulas, N., 2000, ApJ, 534, L75.

Andersson, N. \& Kokkotas, K.D. 2001, Int.J.Mod.Phys., D10, 381.

Bildsten, L. \& Ushomirsky, G., 2000, ApJ,529, L33.

Friedman, J.L. \& Morsink, S.M., 1998, ApJ, 502, 714.

Baym, G., Pethick, C. \& Sutherland, P., 1971, ApJ, 170, 299.

Ghosh, S. K., Phatak, S. C. \& Sahu, K., 1995, Z. Physik A, 352,457. (Proc. Suppl.), 24,110.

Lindblom, L. \& Owen, B. J., 2002, Phys.Rev.D, 65, 063006.

Drago, A., Lavagno, A. \& Pagliara, G., 2005,Phys.Rev.D, 71, 103004.

Nayyar, M. \& Owen, B. J., 2005, astro-ph/0512041.

Haensel, P., Zdunik,J. L. \& Schaeffer, R. 1986, A\&A, 160, 121.

Ipser, J. R. \& Lindblom, L., 1991, ApJ, 373,213.

Kokkotas, K.D. \& Stergioulas, N., 1999, A\&A, 341, 110.

Levin, Y., 1999, ApJ, 517, 328.

Lindblom, L., Mendell, G. \& Owen, B.J., 1999, Phys.Rev.D, 60, 064006.

Lindblom, L., Owen, B.J. \& Ushomirsky, G., 2000, Phys.Rev.D, 62, 084030.

Madsen, J., 1992,Phys.Rev.D, 46, 3290.

Madsen, J., 1998, Phys.Rev.Lett, 81, 3311.

Madsen, J., 2000, Phys.Rev.Lett, 85, 10 
Owen, B. J., 3rd Edoardo Amalai conference on gravitational waves, gr-qc/9912065.

Zheng, X. P., Yang, S. H. \& Li, J. R., 2003,ApJ, 585, L135. 